\title{
Contact tracing in hepatitis B infection
}

\author{
P E MUNDAY,* $\neq$ W MCDONALD,* K M MURRAY-SYKES, † AND J R W HARRIS* \\ From the *Praed Street Clinic, St Mary's Hospital, London; the +Department of Community Medicine, \\ Paddington and North Kensington Health Authority, London; and the $¥$ Sexually Transmitted Diseases \\ Research Group, MRC Clinical Research Centre, Harrow, Middlesex
}

SUMMARY In an epidemiological study of patients diagnosed as having hepatitis B virus (HBV) infection during a 12 month period in a London health district, $98(67 \%)$ of 144 index cases and $146(67 \%)$ of 218 of their named contacts were interviewed. The problems and benefits of using conventional contact tracing techniques in the management of this infection are discussed. Named contacts in stable relationships were more easily traced than young homosexuals with frequent anonymous contacts, and drug addicts. Information on the disease and the risk of its transmission to others was, however, welcomed by some homosexuals who were concerned to establish ways of identifying chronic carriers, immune men, and those at risk. A self help group was started as a result of this study.

\section{Introduction}

For many years the tracing of named contacts of patients with syphilis and gonorrhoea has been regarded as essential for controlling these diseases within the community. In the United Kingdom a well established system exists using health advisers attached to sexually transmitted diseases (STD) clinics. The major role of the health adviser is to identify sexual contacts at risk and to arrange for their examination and treatment, thus interrupting the line of transmission. This approach has been particularly successful in containing syphilis since the long incubation period has facilitated contact tracing. Contact tracing for other STD, particularly in London, has usually been regarded as impracticable on the grounds of cost or inappropriate because no treatment existed for the condition concerned.

Hepatitis B virus (HBV) infection has been recognised as being sexually transmitted since 1973 when Jeffries $e t a^{1}$ and Fulford $e t a^{2}$ reported a high prevalence of infection in patients attending STD clinics. More recent studies have shown that up to $60 \%$ of homosexual men have serological markers of current or previous HBV infection. ${ }^{34}$ Although no treatment is currently available for this condition, the possibility that the chain of transmission might be cut

Address for reprints: Dr P E Munday, Division of Communicable Diseases, Clinical Research Centre, Watford Road, Harrow, Middlesex HA1 3UJ

Accepted for publication 6 April 1983 by contact tracing with a view to modifying behaviour has not been previously explored. The long incubation period of HBV infection would encourage such an approach. Furthermore, the development of a vaccine which may give some protection to exposed persons ${ }^{3}$ and the increasing interest in antiviral drugs ${ }^{5}$ suggest that the possibility of effective treatment is no longer remote.

To study the problems associated with contact tracing of HBV infection, details of all patients who had a positive blood test for hepatitis B surface antigen (HBsAg) resident in a defined geographical area during a designated time were passed to a contact tracer who attempted to interview the patients and trace their contacts.

\section{Patients and methods}

The design of the study will be presented elsewhere. It was approved by the ethical committees of the participating hospitals. In essence, a contact tracer was employed solely to study patients resident, or attending hospitals, in the north west health district of the Kensington and Chelsea and Westminster area health authority between 1 April 1981 and 30 March 1982, who had detectable HBsAg. Patients (index cases) were identified as a result of the positive blood test or were referred on clinical suspicion by general practitioners or hospital doctors. A few homosexual men who heard of the project referred themselves. After obtaining the consent of the doctor responsible for the care of the patient, the contact tracer 
attempted to interview the patient, explain the nature of the project, and enrol him in long term follow up studies. The patient was asked to answer a questionnaire when appropriate dealing with various aspects of his social and sexual behaviour which might be related to HBV transmission. He was also asked to name social and sexual contacts who might have been the source of the infection or secondary contacts and to attend once a month for a blood test if such an investigation were not being conducted elsewhere. Named contacts were then sought and a similar procedure followed. Contacts who were, or who became, HBsAg positive were regarded as index cases. Contacts who were $\mathrm{HBsAg}$ negative were invited to attend monthly during the study period for serological screening.

Serum specimens were tested for $\mathrm{HBsAg}$ by reversed passive haemagglutination (Hepatest, Wellcome Reagents) and confirmed in doubtful cases by radioimmunoassay (Ausria 11-125, Abbott Laboratories). Antibody to hepatitis B surface antigen (anti-HBs) and antibody to hepatitis B core antigen (anti-HBc) were detected by radioimmunoassay (Ausab and Corab, Abbott Laboratories).

\section{Results}

During the study period 144 index cases were identified and $98(68 \%)$ of them were interviewed by the contact tracer. The table shows the reasons for failure to interview. The 98 patients interviewed named 218 contacts, of whom $146(67 \%)$ were interviewed. Serum samples were provided on one or more occasions by all the index cases interviewed and by 122 of the contacts.

TABLE Reasons for failure to interview patients with $\mathrm{HBV}$ infection

Patient refused or failed to cooperate

Hospital refused or failed to cooperate

Inadequate information to permit tracing

Patient left country

General practitioner refused

Language problem

Patient died

Contact tracer failed to reach patient

Total

Of the $81 \mathrm{HBsAg}$ positive patients who submitted more than one serum sample, $39(48 \%)$ became negative during the course of the study. At the first visit nine contacts were already $\mathrm{HBsAg}$ positive and 42 contacts had anti-HBs or anti-HBc. It was not known how long these contacts had been antigen or antibody positive before their recruitment to the study. Three contacts became HBsAg positive and one developed anti-HBs without detectable HBsAg.

\section{Discussion}

Despite the employment of an experienced nurse/ contact tracer who had considerable interpersonal skills and who was occupied solely with the project, it was possible to interview only $98(67 \%)$ of the 144 index cases and $146(67 \%)$ of the 218 named contacts. In addition, a considerable number of anonymous contacts could not be traced. Stable contacts of homosexual men proved relatively easy to study but since these men were generally older they were less likely to be at risk as many already possessed antibody to HBV. Drug addicts were particularly difficult to study since they often had no fixed address and were reluctant to cooperate in long term follow up. Those theoretically most at risk, young homosexual men having frequent anonymous sexual contacts, were impossible to trace using conventional techniques. Furthermore, contact tracing did not identify any chronic carriers of $\mathrm{HBV}$ who were previously unknown, although several index cases were identified who were not receiving appropriate medical care, often because they had no general practitioner.

Nevertheless, provision of a contact tracing service was welcomed by many of the homosexual men who were studied as they found it particularly valuable to discuss their fears in relation to disease in themselves and the risk of transmission to others. Many men had apparently received conflicting advice and some had misunderstood the results of tests. The contact tracer was thus able to clarify some of these points and to advise patients about their potential infectivity, although information about $\varepsilon$ antigen status was available only after the completion of the study.

As the use of conventional techniques for identifying men at risk from anonymous sexual encounters presents an insoluble problem, and as there is considerable concern among articulate and well informed homosexual men about HBV infection, it may be necessary to find novel methods of identifying chronic carriers or those who are at risk. There is already a well established system of nonverbal signs-using arrangements of keys, jewellery, and articles of clothing-to indicate sexual preferences. Some groups of homosexual men are now extending this system to designate chronic carriers, immune men, or those at risk. For example, some men have had $\mathrm{T}$ shirts printed with a logo based on the biohazard warning symbol.

One other beneficial outcome of the study was the establishment of a self help group for chronic HBV carriers to disseminate information to others and to help carriers to come to terms with the implications 
of their infection. This group has recently developed a system of contact lists through which members can get in touch with those whom they will not put at risk-namely, the $60 \%$ of homosexual men who have, or have had, HBV infection.

We thank our many colleagues in general practice, the hospital service, the blood transfusion service, and local authorities who referred patients for the study.

\section{References}

1. Jeffries DJ, James WH, Jefferiss FJG, Macleod KG, Willcox RR. Australia (hepatitis-associated)antigen in patients attending a venereal disease clinic. $B r$ Med $J$ 1973;2:455-6.

2. Fulford KWM, Dane DS, Catterall RD, Woof R, Denning JV Australia antigen and antibody among patients attending a clinic for sexually transmitted diseases. Lancet 1973; i: 1470-3.

3. Szmuness W, Stevens CE, Harley EJ, et al. Hepatitis B vaccine Demonstration of efficacy in a controlled clinic trial in a highrisk population in the United States. N Engl J Med 1980; 303: 833-41.

4. Schreeder MT, Thompson SE, Hadler SC, et al. Hepatitis B in homosexual men: prevalence of infection and factors related to transmission. $J$ Infect Dis 1982;146: 7-15.

5. Dienstag JL, Isselbacher KJ. Therapy for acute and chronic hepatitis. Arch Intern Med 1981; 141:1419-23. 